ORIGINAL ARTICLE

\title{
"Essential noise" - enhancing variability of informational constraints benefits movement control: a comment on Waddington and Adams (2003)
}

\author{
K Davids, R Shuttleworth, C Button, I Renshaw, P Glazier
}

Br J Sports Med 2004;38:601-605. doi: 10.1136/bjsm.2003.007427

See end of article for authors' affiliations

Correspondence to: Keith Davids, School of Physical Education, University of Otago, 55 Union St West, Dunedin, New Zealand; 'kdavids@ pooka.otago.ac.nz

Received

22 September 2003

Accepted for publication

28 September 2003

\begin{abstract}
This commentary proposes a dynamical systems perspective to re-interpret data from a group of international soccer players demonstrating that wearing textured insoles in soccer boots enhanced tactile information from the sole of the foot and increased movement discrimination capacity in ankle inversion sensitivity tests to levels similar to those in barefoot conditions. Theoretical arguments on the functional role of variability induced in the sensorimotor system by textured insoles, acting as a form of "essential noise" to enhance the accuracy of foot positioning are presented. It seems that, far from interfering with motor performance, variability can actually enhance perception of information to support motor performance. The addition of intermittent, intermediate levels of noise in a perceptual motor context may benefit performers by helping them to pick up information signals from background structure. Movement system variability is conceived as noise induced resonance benefiting the pick up of information to regulate behaviour. Variability can be functional in practical programmes to offset negative effects of losses in sensory sensitivity through ageing, disease, illness, or injury
\end{abstract}

$\mathrm{n}$ a recent issue of the British Journal of Sports Medicine Waddington and Adams ${ }^{1}$ presented data from a group of international soccer players showing that: (i) small variations in amplitude of ankle inversion angles were discriminated more easily by players in a barefoot condition than when wearing soccer boots with typical smooth insoles and socks; and (ii) wearing textured insoles (four nodules/ $\mathrm{cm}^{2}$ ) with socks increased the ability to discriminate ankle inversion to levels similar to those in the barefoot condition. In their study 17 players from the Australian women's soccer squad stood astride an ankle movement extent discrimination platform (consisting of a swinging plate rotating along the long axis of the foot under examination), making active inversion movements of one ankle at a time. A psychophysical "absolute judgements" method was used, in which participants were asked to replicate five different ankle inversion extents after a familiarisation process. Waddington and Adams ${ }^{1}$ had some difficulty in explaining their findings, arguing that the added variability of the textured insole surface may have enhanced tactile sensitivity in the feet of the soccer players. However, the mechanism by which a textured insole might enhance tactile sensitivity in tissue at the sole of the foot was not addressed. As they pointed out, from an evolutionary perspective, there is little reason to believe that these data indicate the presence of a vestigial function, available to arboreal ancestors living over 3.5 million years ago, in the non-grasping foot of modern humans.

In this commentary we provide theoretical arguments on the functional role of variability induced in the sensorimotor system by the textured insoles, acting as a form of "essential noise" to enhance the accuracy of foot positioning. The data of Waddington and Adams ${ }^{1}$ may indicate that, relative to the smooth insole, the surface of a textured insole enhances deformation of plantar tissue and provides soccer players with the opportunity to exploit the presence of sensorimotor system noise to enhance perception of haptic information for lower limb positioning. Wearing a sock and smooth insole may dampen noise arising from variable tissue deformation at the sole of the foot, whereas in the textured sole and barefoot condition available background noise may have enhanced the information to control ankle inversion movements. It seems that, far from interfering with motor performance, variability can actually enhance perception of information to support motor performance. A well documented finding in psychophysical experiments of sensory systems is that people rapidly habituate to constant background information, tuning out the signals after short periods of time. ${ }^{2}$ Responses to such signals decline unless variability is increased. As Lackner and Dizio noted, ${ }^{3}$ the mechanisms by which humans use afferent and efferent information to calibrate ongoing movements are only revealed during "exposure to unusual forces and patterns of sensory feedback" (p 279).

A powerful multidisciplinary theoretical framework, dynamical systems theory, encompassing ideas of chaos, complexity, and non-linear dynamics, can account for these findings. From this perspective, variability in movement system behaviour is not seen as typically due to random noise or performance error. Instead it is argued that biological movement systems need access to information to constrain movements, and the presence of noise, in the form of variability of motor output structure, is imperative for functional adaptations to dynamic environments. The counterintuitive message for sports medicine from our commentary is that the addition of intermittent, intermediate levels of noise in a perceptual motor context may benefit performers by helping them to pick up information signals from background structure. To elucidate this argument we begin by providing a brief tutorial of important ideas from dynamical systems theory, continue by examining the relevance of the concept of stochastic resonance in understanding the effects described by Waddington and Adams, ${ }^{1}$ and conclude with a brief discussion of how variability can be functional in practical programmes to offset negative effects of losses in sensory sensitivity through ageing, disease, illness, or injury.

Abbreviations: CoG, centre of gravity; SNR, signal to noise ratio 


\section{INFORMATION-MOVEMENT COUPLING: SIFTING SIGNALS FROM NOISE IN NON-LINEAR DYNAMICAL SYSTEMS}

The capacity to pick up different sources of somatosensory information and couple them to minute adjustments in posture and limb orientation is an important aspect of many everyday human activities, including dynamic sports such as soccer. ${ }^{4}$ The nature of information-movement coupling is of interest to movement scientists seeking to understand how humans coordinate actions in dynamic environments characterised by large amounts of information and background noise. In the study of Waddington and Adams, ${ }^{1}$ it is clear that the problem of detection thresholds for movement sensitivity in the ankle joint is an example of a common problem for humans, that of coupling functional movements to genuine information signals from the background noise present in all complex environments. In understanding information-movement coupling, human movement systems can be considered as belonging to a class of non-linear dynamical systems that are constantly changing and evolving over different timescales. Such systems are composed of several subsystems (for example, hormonal, perceptual, motor), which are typically interacting and engaging in constant energy transactions with the environment. ${ }^{56}$ Kinematic patterns in movement systems are viewed as the product of force (kinetic) fields, which lawfully give rise to flow (informational) fields. The cyclical and mutually inter-dependent relationship between flow and force fields characterises the link between action and perception in functional movement behaviour and is a powerful basis for explaining the interaction of individuals with their environment. ${ }^{7}$ From this viewpoint, perception is ineradicably linked with action so that perceiving event information related to temporal, spatial, and amplitude characteristics, specifies forces and torques required in goal directed movements. ${ }^{8}$ During bipedal standing, for example, haptic information is provided by tissue deformation of muscle, tendinous tissue, and cutaneous receptors adjacent to the soles of the feet, regulating postural control.

An apparent control problem for high dimensional dynamical systems, such as human movement systems, is that they are inherently noisy, demonstrating high levels of variability. Noise has been found at all levels of the human movement system, as revealed by molecular,

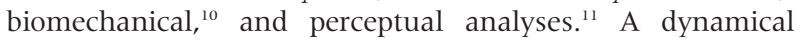
systems theoretical interpretation of variability suggests that noise is an omnipresent and unavoidable characteristic of movement systems. Taken at face value, the findings of Waddington and Adams, ${ }^{1}$ showing that ankle inversion sensitivity was the same in barefoot and textured sole conditions, and better than a smooth sole condition, contradict the traditional "variability as noise or error" hypothesis. However, our commentary suggests that these findings can be understood when one considers how adding so called noise to the sensorimotor system can actually enhance the capacity to pick up information from minute changes in postural regulation movements, to support immediate, functional adaptations in complex and dynamic sport environments.

\section{THE FUNCTIONAL ROLE OF VARIABILITY}

The traditional negative conception of motor system variability as random noise has been based on task outcome measures from behavioural studies (for example, time spent in balance, variation in reaction time measures). ${ }^{11}$ The study of non-linear dynamical systems has revealed that the structure of the variability observed in biological systems over different timescales needs to be carefully understood since it is often functional. ${ }^{11}{ }^{12}$ Healthy and adaptive biological systems depend on variability of behaviour to ensure optimal functioning and an appropriate level of organisational complexity. ${ }^{10}$ Increasing some forms of variability can provide highly functional exploratory behaviour, which reveals useful sources of information to regulate movements. In order to understand how the addition of variability can be functional, one needs to understand the relationship between, noise, randomness, and stochastic processes in movement systems.

\section{THE RELATIONSHIP BETWEEN VARIABILITY, RANDOMNESS, AND STOCHASTISTICITY}

The application of the idea of functional variability to the study of human movement systems emphasises understanding the structure of motor output variability observed. Random processes defy predictions of future states from earlier states since randomness refers to the equiprobability of one event following another event. For this reason, traditionally white noise was the term used to describe the presence of variability with a Gaussian distribution (equiprobable amplitude and frequency changes over time) in sensorimotor systems. In biological systems it has become apparent that noise is not just of the completely random, white Gaussian variety. It is now understood that noise comes in different colours (for example, pink, brown, black), serving different functions. This category of coloured noise is from the $1 / \mathrm{f}$ spectrum and analysis of human movement behaviour is revealing that it can have a complex structure and can influence system behaviour in different ways over different timescales. Thus, noise is typically characterised by degrees of randomness because it is constrained by a variety of factors inherent to the task, individual, and environment. This idea implies that randomness is not the same as variability, since behaviour can be variable and yet deterministic (future events can be influenced by, and therefore, predicted from previous events), for example when changing perceptual information or intentions constrain movement system behaviour in different ways. In reality, a range of deterministic and variable processes contributes to observed fluctuations in movement and its outcomes. ${ }^{13}$ Riley and Turvey ${ }^{14}$ described a process called "piecewise determinism" in which particular combinations of variable and deterministic behaviour emerge as humans attempt to satisfy different task constraints by allowing variability in redundant biomechanical degrees of freedom whilst in other parts of the motor system it is minimised. Functional blending of variable and deterministic behaviour comes about as each individual performer, with different intentions and perceptions, strives to satisfy the constraints on him/her. The implication of these ideas is that each individual performer can exploit variability within the movement system in different ways to adapt to changing task constraints over time. ${ }^{13}$

These ideas have been supported by recent work on the relationship between perceptual information and postural control reviewed by Riley and Turvey. ${ }^{14}$ When individuals try to maintain upright stance with eyes open and closed, their behaviour (evidenced by postural sway characteristics) changes accordingly. With eyes open, postural sway becomes less variable and comprises more randomness. With eyes closed, postural sway becomes less random, but behaviour comprises more deterministic variability. Individuals exploit motor system variability with eyes closed by using postural control musculature to create haptic information in order to perceptually regulate upright stance, that is, make postural behaviour more deterministic. Clearly, the presence of different coloured noise in movement systems underlines why variability cannot be equated with complete randomness and Riley and Turvey ${ }^{14}$ argued that "More variable does not mean more random, and more controllable does not mean 
more deterministic"(p 100). Similar findings have been found when individuals were allowed to use haptic information from a light finger touch of an environmental surface during upright stance without vision. The strategy of increasing the amount of postural activity when visual perceptual information is degraded or constrained is a healthy biological system's way of reducing the influence of random fluctuations in the postural control subsystem, and aids in the detection and pick up of proprioceptive information in postural muscles.

These findings are relevant for our commentary on the data of Waddington and Adams ${ }^{1}$ because they suggest that increasing variability may be the mechanism by which textured insoles support the same level of performance on ankle inversion discrimination tests as barefoot conditions. The idea of individuals benefiting from an increased amount of variability in movements or tasks, to enhance stability of performance, is counterintuitive to traditional notions of variability as completely random noise or error in movement systems. ${ }^{11}$ The structure of motor output variability needs to be carefully understood before accurate inferences can be drawn about the variability observed in movement behaviour by sports clinicians and sport scientists. In some parts of the system, higher levels of variability can actually reflect non-conscious compensatory measures by individuals. ${ }^{5}$ Furthermore, as we note below, other theoretical ideas from the study of non-linear dynamical systems are contributing to our understanding of the message that variability in the sensorimotor system can be functional.

\section{STOCHASTIC RESONANCE}

With respect to the findings of Waddington and Adams, another important feature of non-linear dynamical systems is stochastic resonance. From an engineering and computer science perspective, it has been known for some time that the presence of noise may often be beneficial in the pick up of weak or diffuse signals to support system performance. Stochastic resonance is an effect also found in many nonlinear systems allowing information signals surrounded by background noise to be transmitted without being highly degraded, through the simple mechanism of adding an intermediate level of background noise. It has been defined as a noise induced rise in the signal to noise ratio (SNR) of an information signal within a non-linear system. ${ }^{15}$ As we noted earlier, it is a mistake to equate stochasticity with complete randomness since stochastic processes in biological movement systems vary in the contribution of random and deterministic components. ${ }^{14}$ While in linear systems the SNR decreases with increasing noise intensity, ${ }^{15}$ in nonlinear systems noise is not additive and its presence can often increase the SNR at output. Stochastic resonance works by lowering the SNR degradation that occurs when a signal is transmitted through a non-linear system, and responses to signals within certain frequency ranges can be greatly enhanced through the influence of intermediate levels of noise.

Since its discovery in the early 1980s in physical systems, the role of stochastic resonance has been clarified in many biological systems including fish, insects, and humans. In recent years it has been shown that interaction between noise and signal can improve human sensorimotor integration. ${ }^{16}$ Recently, a double receptor design (noise and signals were injected into two different receptors in the brain) was used to demonstrate the behavioural consequences of stochastic resonance. ${ }^{17}$ Humans performed a sensorimotor integration task in response to a slowly changing level of colour (grey) perceived. Using a mirror stereoscope, a weak-signal visual stimulus was presented to the right eye and random stimuli to the left eye. Handgrip response was used to quantify the way that higher visual centres in the brain supported perception of signal and noise. For many participants, cross correlations of grip modulation and presence of noise were almost zero at low levels of noise, increased for intermediate levels of noise, and decreased to zero again for high levels of noise. Analyses ${ }^{17}$ revealed that "behavioral driving by subthreshold signals can be improved by adding noise with an SD ranging from a half to twice that of the signal amplitude..." (p 218106). The conclusion was that improved performance might have been associated with an increased synchrony of neural activity in appropriate sensorimotor areas of the human cortex. ${ }^{17}$

The role of stochastic resonance has also been investigated in human sensorimotor systems during postural control tasks. This idea was exemplified in a study of young and elderly adults by Priplata and colleagues $^{18}$ who were interested in whether postural control could be enhanced by the application of subsensory mechanical noise to the feet. Actuators placed under a platform interfacing with the soles of participants' feet during quiet standing drove displacement forces in indentors. White noise signals low pass filtered to $100 \mathrm{~Hz}$ were used in the experiment. Mean square radial displacement plots over time were calculated along with more traditional measures of postural sway, under control and added noise conditions. Within individual comparisons showed beneficial effects of mechanically applied noise in reducing amounts of postural sway and enhancing feedback, through the mechanism of negative masking and the addition of stochastic resonance, for both older and younger adults. But it was also clear that all individuals exhibited functional postural sway in short time scales around longer term background activity in postural control muscles, indicative of exploratory behaviour of the postural control system, when noise was applied to the platform. Of relevance to the findings of Waddington and Adams, ${ }^{1}$ Priplata and coworkers ${ }^{18}$ proposed that their data showed that application of subthreshold noise acted as a "suitable pedestal for enhancing the detection of pressure changes on the sole of the feet" (p 814).

The data from these studies of human sensorimotor integration imply that, when making sensitive, psychophysical judgements of foot position, as required by Waddington and Adams, ${ }^{1}$ the noise provided by a textured insole improved SNR, allowing players to discriminate subtle differences in ankle inversion more effectively than the smooth insole (fig 1).

\section{IMPLICATIONS FOR SPORTS MEDICINE}

These ideas have a number of important implications for clinicians, therapists, coaches, and sport scientists interested in rehabilitation and preparation of athletes for performance. First, the addition of noise or variability in practice and rehabilitation sessions may enhance the capacity of a performer to pick up signals from background noise, a vital task in sport practice, training, and rehabilitation from injury. There are a variety of ways in which these ideas can be implemented in therapeutic programmes with different population groups such as ageing, disabled, injured athletes, and young children. ${ }^{5}$ For example, an implicit assumption of traditional research on postural control is that postural instability is associated with large and highly variable sway oscillations. However, studies conducted under the framework of non-linear dynamical systems theory suggest that, under certain task constraints, healthy individuals often exhibit greater sway oscillations than other individuals, including older adults and Parkinson patients. ${ }^{11}$ Furthermore, the data of Priplata and colleagues $^{18}$ on stochastic resonance suggested that artificial aids such as randomly vibrating shoe insoles could attenuate postural 


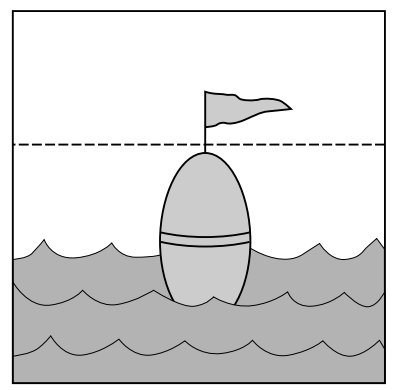

Barefoot condition

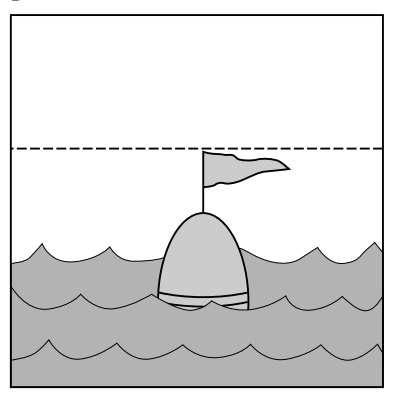

Smooth insole
C

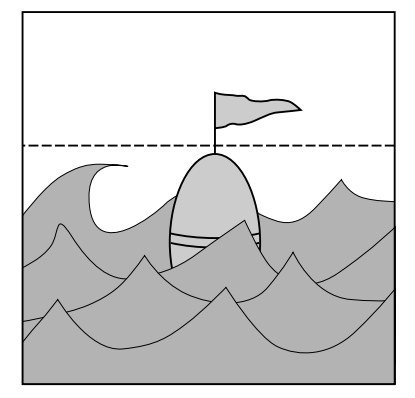

Textured insole

Figure 1 Pictorial explanation of noise induced resonance. The perception of sensory information concerning ankle inversion extent in Waddington and Adam's study' is depicted as a set of three images to demonstrate the concept of noise induced resonance. In each figure, the buoy represents the signal (that is, tactile and proprioceptive feedback from the sole of the foot). The dotted line represents the stimulus threshold (that is, when the flag is below the line the stimulus cannot be detected by the participant, when the flag is above the line the sensory information is detectable). Finally the fluid in which the buoy is partly submerged represents background noise that is always present to some extent in natural environments (for example, distracting information or fatigue). In the barefoot condition (A), the signal is clearly detectable as there is no dampening effect of either socks or insoles. There is a small amount of background noise that has little effect on the SNR. When the participants wear a smooth insole (B), the signal is now less clear. The introduction of socks and boots has dampened the signal (for example, submerged the signal in background noise). Whilst there has been no added noise, the SNR has decreased. The textured insole (C) induces noise into the system (for example, the fluid's waves are higher). Whilst there is a small dampening effect of the socks, the signal is enhanced by noise induced by tissue deformation in the textured insoles, creating the resonance effect. In this case, additional background noise is functional returning the system to similar levels of perception as in the barefoot condition.

sway in older adults, approximating values associated with younger adults, to counteract age related deficits in balance control. Since sway variability is rarely random, understanding of the structure of noise in postural control systems will reveal how these apparently contradictory findings can be understood in non-linear dynamics. High frequency postural fluctuations under short timescales could indicate exploratory behaviour by individuals to reveal information, whereas low frequency fluctuations signify long range correlations or deterministic behaviour constrained by intentions or motor system constraints (for example, induced by ageing effects or injury). The use of textured insoles in sports shoes may be a way of enhancing the perception of haptic information signals relative to other signals or background noise present in a neural system (fig 1). Counterintuitively, the efficacy of sports medicine may be enhanced if practitioners carefully considered ways of increasing variability of informational and task constraints faced by patients during rehabilitation practices. Indeed, Strickland and colleagues ${ }^{19}$ found that increasing the range of movement around the ankle joint (that is, changing the intrinsic dynamics of the individual's movement system) through exposure to a proprioceptive neuromuscular facilitation session resulted in athletes producing greater anterio-posterior postural sway. This type of task constrained variability can be indicative of increasing functional postural sway leading to exploratory (variable but deterministic) behaviour in order to detect the centre of gravity (CoG), important for maintaining "good" balance during everyday behaviour as well as high level sport performance. Often a performer's inability to detect changes in stance during action can lead to performance decrements. For example, when waiting in the "reception" position to respond to a shot in soccer, having the CoG too far forward could lead to a goalkeeper being unable to respond to a ball played behind him. Conversely, having the CoG too far "back" could lead to failure to reach forward to a shot bouncing in front.

Second, these findings in sports medicine can be associated with recent advances in the use of information technology to enhance intrinsic feedback mechanisms for athletes. High performance coaches in gymnastics and pistol shooting are now able to access augmented feedback technologies that sensitise performers to proprioceptive information in order to enhance balance and stability, and these ideas could transfer to clinical rehabilitation programmes with a range of individuals. ${ }^{20}$ For example, vibrating inserts in handles of implements such as rackets, bats, and clubs, may enhance grip force coordination to resist load forces when intercepting projectiles in sport, during recovery from wrist and lower arm injuries. The findings of Waddington and Adams ${ }^{1}$ imply that manipulating informational and task constraints to introduce low levels of noise into the system might be used to develop increased sensitivity to proprioceptive information, crucial to success in many sports.

In summary, considering human movement systems as open, non-linear dynamical systems suggests that variability may have a functional role, as an essential form of noise, in helping individuals explore their environment to pick up information for actions. At many different levels of analysis, from the study of molecular and cellular function to analysis of effects of gene pool diversity in behavioural genetics, the fundamental role of variability in the adaptive behaviour of biological systems is becoming obvious. The implication for sports medicine is that variability in movement should not be equated with completely random behaviour, and clinicians need to interpret carefully the nature (that is, the structure and function) of noise observed in movements. A final point to note is that we have argued that the term stochastic resonance may be somewhat misleading without a clear understanding of the nature of stochastic processes in biological movement systems. A better term to describe the functional effects of the variability observed in adaptive movement behaviour may be noise induced resonance to denote that stochastic processes are composed of a blend of deterministic and random effects. ${ }^{14}$ Further research is needed to explore how stochastic or noise induced resonance can be used to enhance the quality of training, practice, and rehabilitation programmes in sport medicine and sport science.

\section{ACKNOWLEDGEMENTS}

The authors would like to acknowledge the help of Maureen Hazelwood in the preparation of this manuscript and Chris Sullivan for artwork. 


\section{What is already known}

Stochastic resonance has been studied in many physical and biological systems and evidence has emerged to support its beneficial role in supporting functional behaviour. It has rarely been applied to human behaviour, and its relevance to sport and exercise science and sports medicine is little understood.

\section{What this study adds}

This study draws attention to the beneficial effects of textured insoles on movement control. A convincing explanation for the mechanism for previous findings was lacking. This commentary provides such a theoretical rationale and raises the awareness of sports medicine specialists to the potential value of functional noise or variability in training and therapeutic programmes.

\section{Authors' affiliations}

K Davids, R Shuttleworth, C Button, School of Physical Education, University of Otago, Dunedin, New Zealand

I Renshaw, Division of Sport and Recreation, Auckland University of Technology, Auckland, New Zealand

P Glazier, School of Sport, Physical Education and Recreation, University of Wales Institute at Cardiff, Cardiff, Wales, UK

Conflict of interest: none declared.

\section{REFERENCES}

1 Waddington G, Adams R. Football boot insoles and sensitivity to extent of ankle inversion movement. Br J Sports Med 2003;37:170-5.
2 Givois V, Pollack GS. Sensory habituation of auditory receptor neurons: implications for sound localization. J Exp Biol 2000;203:2529-37.

3 Lackner JR, Dizio PA. Aspects of body self-calibration. Trends Cogn Sci 2000;6:279-88.

4 Davids K, Lees A, Burwitz L. Understanding and measuring coordination and control in soccer skills: implications for talent identification and skill acquisition. J Sports Sci 2000;18:703-14.

5 Davids K, Button C, Bennett SJ. Acquiring movement skill: a constraints-led perspective. Champaign, IL: Human Kinetics, in press.

6 Kelso JAS. Dynamic patterns: the self-organisation of brain and behaviour. Cambridge, MA: MIT Press, 1995.

7 Gibson JJ. The ecological approach to visual perception. Hillsdale, NJ: Lawrence Erlbaum Associates, 1979.

8 Turvey MT, RE Shaw. Toward an ecological physics and a physical psychology. In: Solso RL, Massaro DW, eds. The science of the mind: 2001 and beyond. New York: Oxford University Press, 1995: 144-69.

9 Gardner TS, Collins JJ. Neutralizing noise in gene networks. Nature 2000:405:520-1.

10 van Emmerik REA, van Wegen EEH. On variability and stability in human movement. J Appl Biomech 2000;16:394-406

11 Davids K, Glazier P, Araújo D, et al. Movement systems as dynamical systems: the role of functional variability and its implications for sports medicine. Sports Med 2003;33:245-60.

12 Collins JJ. Fishing for function in noise. Nature 1999;402:241-2.

13 Newell KM, Slifkin AB. The nature of movement variability. In: Piek JP, ed. Motor behavior and human skill: a multidisciplinary perspective. Champaign, IL: Human Kinetics, 1998:143-60.

14 Riley MA, Turvey MT. Variability and determinism in motor behavior. J Motor Behav 2002;34:99-125.

15 Dykman MI, McClintock PVE. What can stochastic resonance do? Nature 1998;391:344.

16 Usher $M$, Feingold $M$. Stochastic resonance in the speed of memory retrieval. Biol Cybern 2000;83:1 1-6.

17 Kitajo K, Nozaki D, Ward L, et al. Behavioral stochastic resonance within the human brain. Phys Rev Lett 2003;90:218103-7.

18 Priplata A, Niemi J, Salen M, et al. Noise-enhanced human balance control. Phys Rev Lett 2002;89:238101-4.

19 Strickland J, Price M, Goss-Sampson M. The sit-and-reach test: just what are we measuring? J Sport Sci 2003;21:307-8.

20 Liebermann DG, Katz L, Hughes MD, et al. Advances in the application of information technology to sport performance. J Sport Sci 2002;20:755-69. 